\title{
SEISMOLOGY OF A MASSIVE PULSATING HYDROGEN ATMOSPHERE WHITE DWARF
}

\author{
S. O. Kepler ${ }^{1}$, Ingrid Pelisoli ${ }^{1}$, Viviane Peçanha ${ }^{1}$, J. E. S. Costa ${ }^{1}$, Luciano Fraga ${ }^{2}$, J. J. Hermes ${ }^{3}$, D. E. Winget ${ }^{3}$, \\ Barbara Castanheira $^{3,4}$, A. H. Córsico ${ }^{5}$, A. D. Romero ${ }^{5}$, Leandro Althaus ${ }^{5}$, S. J. Kleinman ${ }^{6}$, A. Nitta ${ }^{6}$, \\ D. Koester ${ }^{7}$, Baybars Külebi ${ }^{8}$, Stefan Jordan $^{9}$, And Antonio KanaAn ${ }^{10}$ \\ ${ }^{1}$ Instituto de Física, Universidade Federal do Rio Grande do Sul, 91501-970 Porto Alegre, RS, Brazil; kepler@ @if.ufrgs.br \\ ${ }^{2}$ Southern Observatory for Astrophysical Research, Casilla 603, La Serena, Chile \\ ${ }^{3}$ Department of Astronomy and McDonald Observatory, University of Texas, Austin, TX 78712-1083, USA \\ ${ }^{4}$ Institut für Astronomie, Türkenschanzstr. 17, A-1180 Wien, Austria \\ ${ }^{5}$ Facultad de Ciencias Astronómicas y Geofísicas, Universidad Nacional de La Plata, Argentina \\ ${ }^{6}$ Gemini Observatory, Northern Operations Center, 670 North A'ohoku Place, Hilo, HI 96720, USA \\ ${ }^{7}$ Institut für Theoretische Physik und Astrophysik, Universität Kiel, D-24098 Kiel, Germany \\ ${ }^{8}$ Institut de Ciències de L'Espai, Universitat Autònoma de Barcelon and Institute for Space Studies of Catalonia, c/Gran Capità 2-4, \\ Edif. Nexus 104, E-08034 Barcelona, Spain \\ ${ }_{9}^{9}$ Astronomisches Rechen-Institut, ZAH, Mönchhofstr. 12-14, D-69120 Heidelberg, Germany \\ ${ }^{10}$ Departamento de Física, Universidade Federal de Santa Catarina, Florianópolis, SC, Brazil \\ Received 2012 June 23; accepted 2012 August 8; published 2012 September 17
}

\begin{abstract}
We report our observations of the new pulsating hydrogen atmosphere white dwarf SDSS J132350.28+010304.22. We discovered periodic photometric variations in frequency and amplitude that are commensurate with nonradial g-mode pulsations in ZZ Ceti stars. This, along with estimates for the star's temperature and gravity, establishes it as a massive ZZ Ceti star. We used time-series photometric observations with the $4.1 \mathrm{~m}$ SOAR Telescope, complemented by contemporary McDonald Observatory $2.1 \mathrm{~m}$ data, to discover the photometric variability. The light curve of SDSS J132350.28+010304.22 shows at least nine detectable frequencies. We used these frequencies to make an asteroseismic determination of the total mass and effective temperature of the star: $M_{\star}=0.88 \pm 0.02 M_{\odot}$ and $T_{\text {eff }}=12,100 \pm 140 \mathrm{~K}$. These values are consistent with those derived from the optical spectra and photometric colors.
\end{abstract}

Key words: stars: fundamental parameters - stars: oscillations - white dwarfs

Online-only material: color figures

\section{INTRODUCTION}

Roughly $97 \%$ of all stars born have main-sequence masses less than $8-10 M_{\odot}$; these stars will evolve to become white dwarf stars (e.g., Weidemann 2000). This is in agreement with Podsiadlowski et al. (2004), who estimate $11 \pm 1 M_{\odot}$ as the lower limit for stars that evolve to neutron stars. White dwarf stars are therefore records of stellar evolution history (Fontaine \& Brassard 2008; Winget \& Kepler 2008; Althaus et al. 2010b). Because of their compact nature and high internal temperatures, they are also useful as probes of high energy density physics (e.g., Kepler et al. 2005; Isern et al. 2010). Spectroscopically, more than $80 \%$ of all white dwarf stars show only hydrogen lines and are classified as spectral type DA (e.g., Kepler et al. 2007; Bergeron et al. 2011). As DAs cool below an effective temperature $\sim 13,000 \mathrm{~K}$, they develop a partial ionization zone near the surface that results in surface convection. As the convection zone becomes deep enough, near $12,200 \mathrm{~K}$, depending on the mass of the star, most if not all DA white dwarf stars start to pulsate in non-radial g-mode pulsations (e.g., Castanheira et al. 2010). We report the discovery of pulsations in the higher than average mass DA SDSS J132350.28+010304.22.

We fit the two existing Sloan Digital Sky Survey (SDSS) spectra of this star with synthetic spectra, based on atmosphere models, using ML2 $/ \alpha=0.6$ (e.g., Bergeron et al. 1995) mixing length convection parameterization, and Tremblay et al. (2010) non-ideal Stark broadening (Koester et al. 2009; Koester 2010). Each SDSS spectra can be uniquely identified by a
Plate-Modified Julian Date-Fiber number. The two spectra, with Plate-Modified Julian Date-Fiber $=0297-51959-332$ and $\mathrm{S} / \mathrm{N}=$ 14 , yield a fit of $T_{\text {eff }}=11,780 \pm 160 \mathrm{~K}$ and $\log g=8.56 \pm 0.06$, and $M=0.94 \pm 0.03 M_{\odot}$ (Figure 1), while 0297-51663-324, with $\mathrm{S} / \mathrm{N}=11$, yields a fit of $T_{\text {eff }}=11,900 \pm 230 \mathrm{~K}$ and $\log g=8.45 \pm 0.08$, and $M=0.88 \pm 0.05 M_{\odot}$; the masses were obtained using the evolutionary models of Althaus et al. (2010a). Tremblay et al. (2011) independently fit the same SDSS spectra with their $\alpha=0.8$ models and find similar values: $T_{\text {eff }}=11,680 \pm 200 \mathrm{~K}$ and $\log g=8.59 \pm 0.07$, and a mass $M=0.98 \pm 0.05 M_{\odot}$.

Castanheira et al. (2010) discuss the 148 DAVs known to date and none have reported magnetic field determinations. Wickramasinghe \& Ferrario (2005) quote a mean mass of $0.93 M_{\odot}$ for magnetic white dwarfs, compared with $0.6 M_{\odot}$ for normal white dwarfs, based on Liebert et al. (2003) determinations. As magnetic field broadening of the lines can be mistaken as higher gravity, we need to determine if the mass estimated from the spectra is affected by a magnetic field.

We report higher signal-to-noise $(\mathrm{S} / \mathrm{N})$ spectra and polarization measurements showing no clear evidence that SDSS $\mathrm{J} 132350.28+010304.22$ is magnetic to our detection limit around $1 \mathrm{MG}$.

\section{OBSERVATIONS: PHOTOMETRIC AND SPECTROSCOPIC}

We obtained time series of $30 \mathrm{~s}$ images with the B filter on the night of 2011 April 28, using the SOAR Optical Imager attached to the SOAR $4.1 \mathrm{~m}$ telescope, detecting ZZ Ceti-type pulsations 


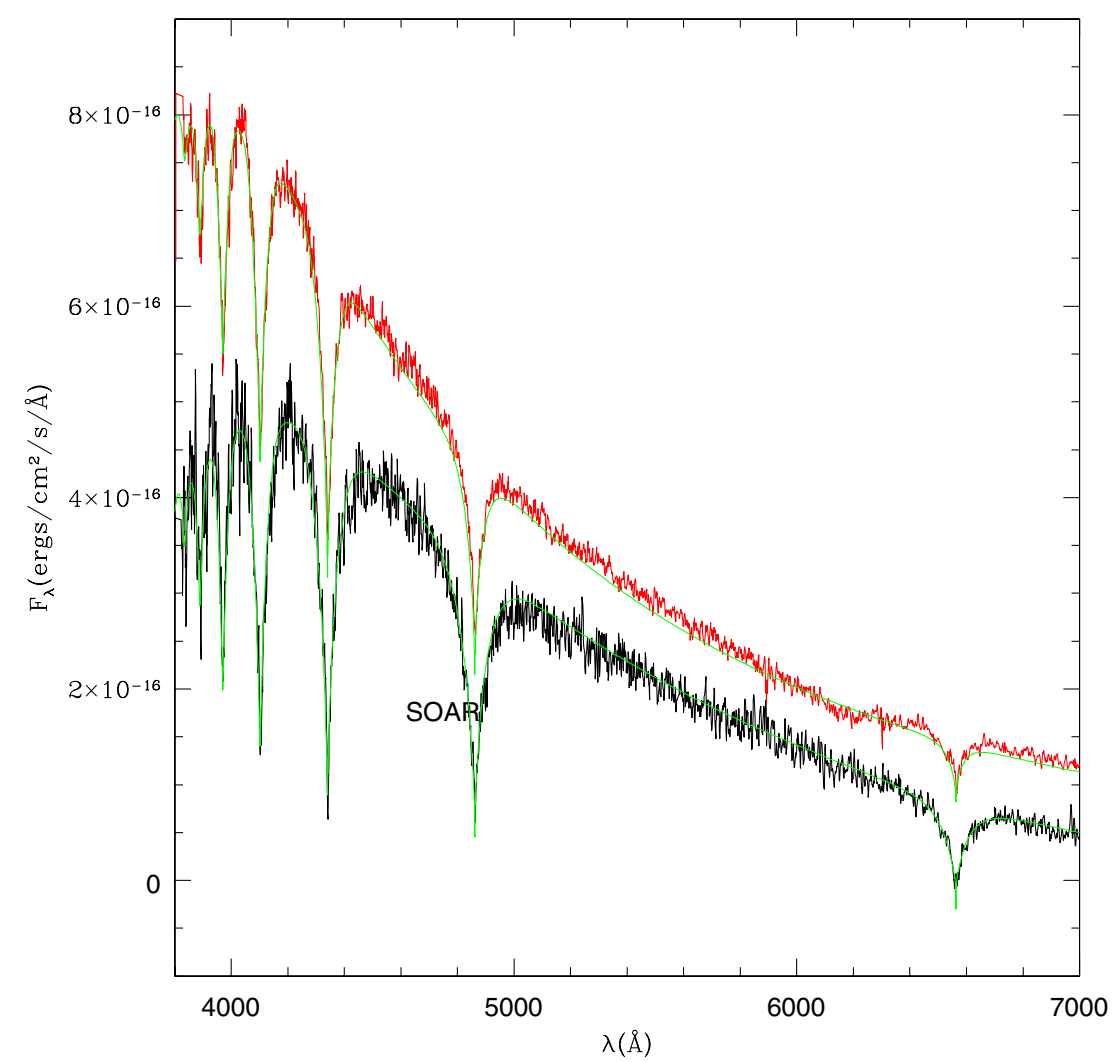

Figure 1. Highest $\mathrm{S} / \mathrm{N}$ spectra, $\mathrm{PMF}=0297-51959-332$, spectra from SDSS (upper: red) and Soar (lower: black).

(A color version of this figure is available in the online journal.)

Table 1

Multisinusoidal Fit

\begin{tabular}{lccc}
\hline \hline$f_{i}$ & $\begin{array}{c}\text { Frequency } \\
(\mu \mathrm{Hz})\end{array}$ & $\begin{array}{c}\text { Period } \\
(\mathrm{s})\end{array}$ & $\begin{array}{c}\text { Amplitude } \\
(\mathrm{mma})\end{array}$ \\
\hline$f_{1}$ & $1633.37 \pm 0.05$ & $612.23 \pm 0.02$ & $11.9 \pm 0.7$ \\
$f_{2}$ & $1818.83 \pm 0.06$ & $549.81 \pm 0.02$ & $6.7 \pm 0.7$ \\
$f_{3}$ & $1694.54 \pm 0.08$ & $590.13 \pm 0.03$ & $7.1 \pm 0.7$ \\
$f_{4}$ & $1571.37 \pm 0.12$ & $636.39 \pm 0.05$ & $4.8 \pm 0.7$ \\
$f_{5}$ & $1431.35 \pm 0.10$ & $698.64 \pm 0.05$ & $4.3 \pm 0.7$ \\
$f_{6}$ & $1490.18 \pm 0.11$ & $671.06 \pm 0.05$ & $4.4 \pm 0.7$ \\
$f_{7}$ & $1203.28 \pm 0.10$ & $831.06 \pm 0.07$ & $4.6 \pm 0.7$ \\
$f_{8}$ & $2018.39 \pm 0.10$ & $495.44 \pm 0.03$ & $4.1 \pm 0.7$ \\
$f_{9}$ & $1131.00 \pm 0.11$ & $884.17 \pm 0.09$ & $4.1 \pm 0.7$ \\
\hline
\end{tabular}

in SDSS J132350.28+010304.22 for the first time. We obtained follow-up observations at SOAR on the night of 2011 May 6 and contemporaneously at the McDonald $2.1 \mathrm{~m}$ Struve telescope using the Argos prime focus camera on the nights of 2011 May 6 and 7, with 15 s exposures, and a BG 40 filter, confirming this object as a new DAV (Figure 2). The standard reduction procedures and weighted aperture photometry of a total of $600 \times 30 \mathrm{~s}$ and $1584 \times 15 \mathrm{~s}$ images were performed as described in Kanaan et al. (2000). The Fourier transform of combined two-site light curve (Figure 3) shows nine periodicities (Table 1) to a $99.9 \%$ confidence level of 3.4 millimodulation amplitude (mma). The confidence level was calculated by randomizing the light curve as described by Costa et al. (1999).

We have also carried out spectroscopic observations using the SOAR Goodman High Throughput Spectrograph to refine our spectroscopic mass determinations. The spectrograph was configured with a 3 arcsec wide slit and using a volume phase holographic $12001 \mathrm{~mm}^{-1}$ grating centered around $\mathrm{H} \alpha$ on the night of 2011 May 12, and using a $21001 \mathrm{~mm}^{-1}$ grating around $\mathrm{H} \beta$ on the nights of 2011 June $1-3$. The spectroscopic reduction procedures of a total of $3 \times 1800 \mathrm{~s}$ around $\mathrm{H} \alpha, 6 \times 1224 \mathrm{~s}$ and $9 \times 612 \mathrm{~s}$, both around $\mathrm{H} \beta$, were performed using standard IRAF routines, which include: bias subtraction, flat-fielding, cosmic-ray cleaning, and flux and wavelength calibration. The combined spectrum shown in Figure 4 has an $S / N$ of $\simeq 20$.

In an attempt to search for magnetic fields, we obtained spectropolarimetry with FORS2 on ESO's Very Large Telescope (VLT), using the GRISM $300 \mathrm{~V}+10$ setup and the $\lambda / 2$ retarder plate, on June 30 and July 1 . We acquired $4 \times 1500 \mathrm{~s}$ exposures with the $\pm 45 \mathrm{deg}$ circular polarizer setup. Figure 5 shows the spectropolarimetric measurements, showing no evidence of magnetic fields, but with a low $\mathrm{S} / \mathrm{N}$ in Stokes $V / I$. Figure 6 follows the low-field approach of Bagnulo et al. (2002, 2012) to test for a magnetic field. The lack of significant inclination of the line is consistent with zero longitudinal field. We also detect no quadratic Zeeman displacements of the line centers, even for the higher Balmer lines.

\section{RESULTS}

Schmidt \& Smith (1995) discuss that circular polarization cannot be detected for equator on line-of-sight fields, and we used their formula to estimate the probability of not detecting a field due to inclination, $p=2.5 B_{\lim .} / B_{d}=50 \%$ for $B_{\text {lim. }}=200 \mathrm{kG}$ and $B_{d}=1 \mathrm{MG}$ (Figure 5). To test for a magnetic field with the equatorial plane close to the line of sight, it will be necessary to measure linear polarization. 

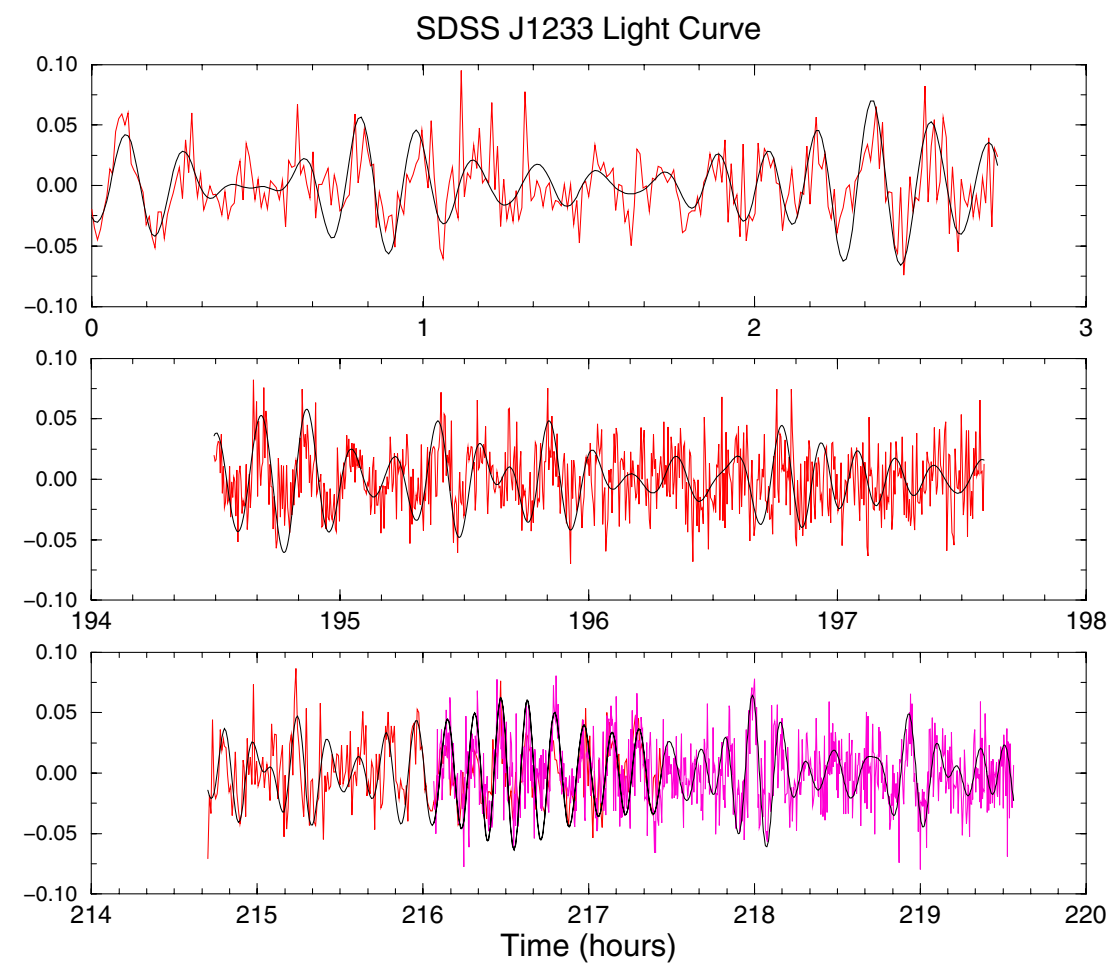

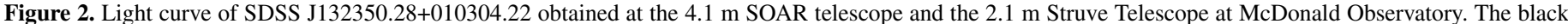
line shows the sum of nine sinusoids with the amplitude and phases obtained from a nonlinear least-squares fit to the whole time series. (A color version of this figure is available in the online journal.)

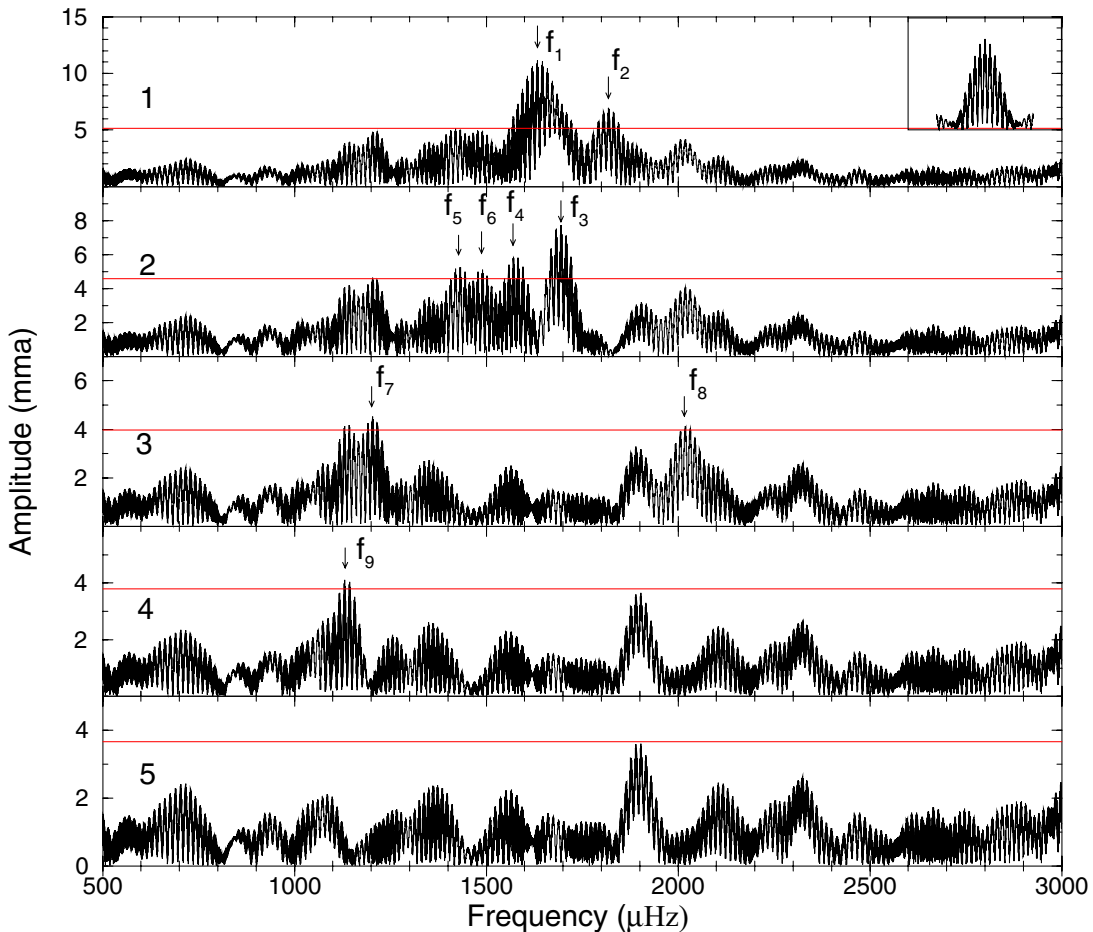

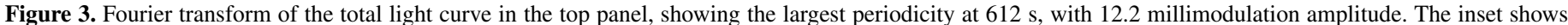

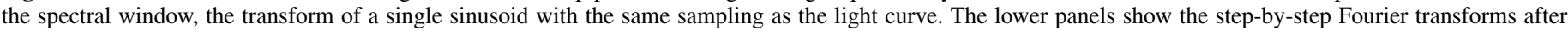

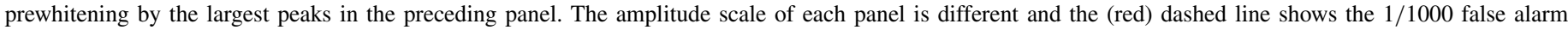
probability estimated for that panel.

(A color version of this figure is available in the online journal.)

The high S/N ESO spectra, also shown in Figure 5, fit a lower temperature, $T_{\text {eff }}=11,300 \pm 50 \mathrm{~K}, \log g=8.73 \pm 0.02$, and $M=1.049 \pm 0.011 M_{\odot}$, if we fit the whole spectra, but $T_{\text {eff }}=11,980 \pm 10 \mathrm{~K}, \log g=8.56 \pm 0.01$, and $M=$
$0.956 \pm 0.005 M_{\odot}$, if we fit only the line profiles. The line profile fit is more reliable because the flux calibration did not include the retarder plate or a redetermination of the extinction after the 2011 Puyehue volcano explosion. The 


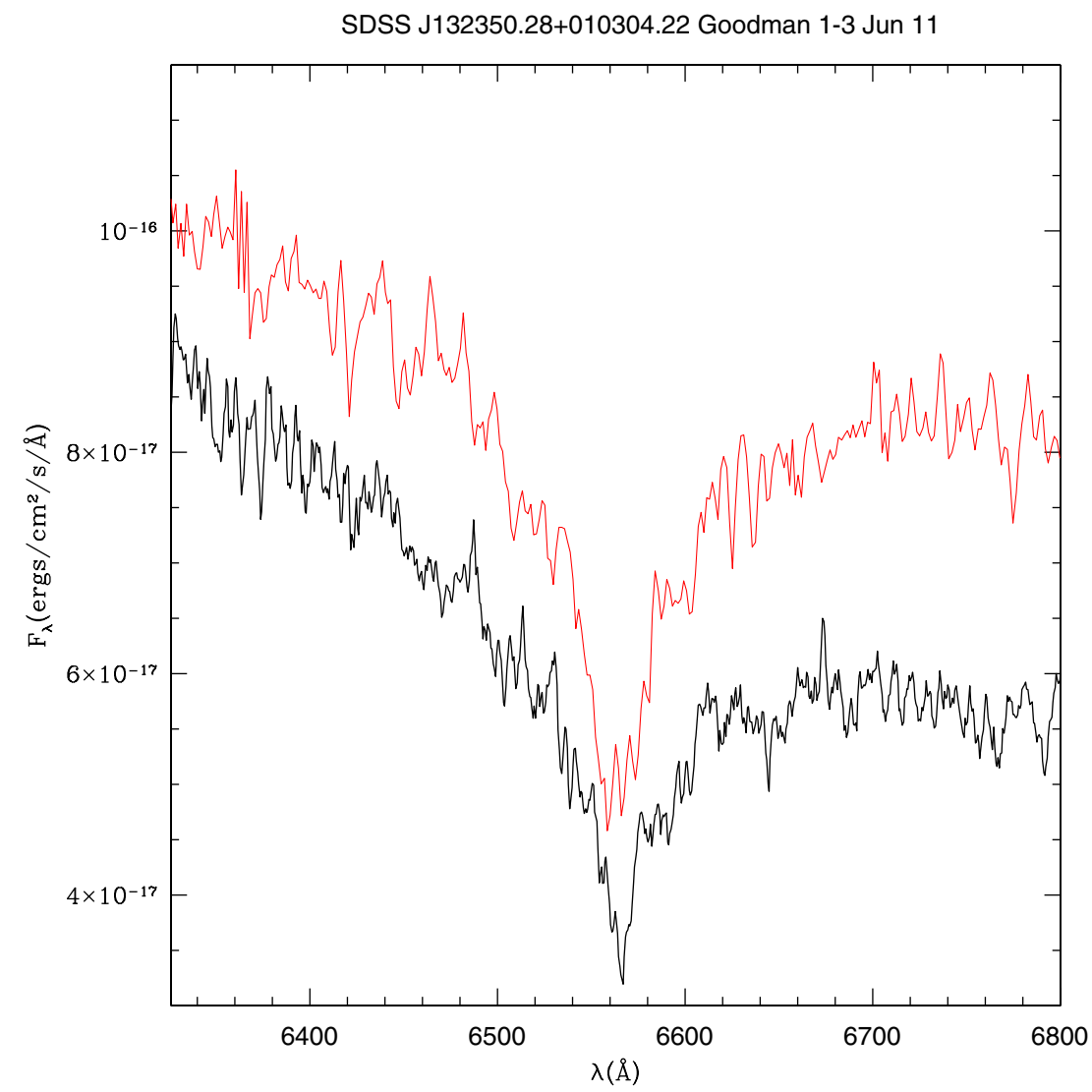

Figure 4. Spectra from SOAR 4.1 m telescope around $\mathrm{H} \alpha$. The upper line shows the PMF = 0297-51959-332 SDSS spectrum. (A color version of this figure is available in the online journal.)

Table 2

Seismological Fit with the LAPLATA Models

\begin{tabular}{lcccc}
\hline \hline$P_{\text {obs }}$ & $P_{\text {theo }}$ & $\ell$ & $k$ & $\delta$ \\
\hline 612.23 & 610.35 & 1 & 14 & 1.88 \\
589.68 & 591.70 & 2 & 25 & 2.02 \\
549.80 & 551.80 & 2 & 23 & 2.00 \\
636.32 & 636.02 & 2 & 27 & 0.30 \\
704.34 & 704.00 & 1 & 17 & 0.34 \\
671.05 & 671.46 & 1 & 16 & 0.41 \\
839.14 & 832.59 & 2 & 36 & 6.56 \\
495.43 & 490.59 & 1 & 11 & 4.88 \\
884.18 & 885.10 & 1 & 22 & 0.92 \\
\hline
\end{tabular}

low-resolution spectrum shows no evidence of a magnetic field.

Assuming that the nine periodicities detected in the light curve are normal g-modes, we made an amplitude-weighted fit of the observed periods to those computed from the full evolutionary models of Althaus et al. (2010a), the LAPLATA models, following Romero et al. (2012). The results of seismology are: $M_{*}=0.88 \pm 0.02 M_{\odot}, T_{\text {eff }}=12,100 \pm 140 \mathrm{~K}, M_{\mathrm{H}}=(4.0 \pm$ $3.3) \times 10^{-7} M_{*}, M_{\mathrm{He}}=(2.6 \pm 0.3) \times 10^{-3} M_{*}, \log \left(L / L_{\odot}\right)=$ $-2.79 \pm 0.05, \log \left(R / R_{\odot}\right)=-2.038 \pm 0.025, \log g=8.46 \pm$ $0.07, X_{C}=0.37 \pm 0.01, X_{O}=0.62 \pm 0.01$, progenitor mass $M_{\text {initial }}=5.0 \pm 0.5 M_{\odot}$, and $\phi=2.15 \mathrm{~s}$, the average of the differences between theoretical and observed periods (Table 2). We estimate that the uncertainties in the physics, such as the uncertainty in the cross section of the $\mathrm{C}(\alpha, \gamma) \mathrm{O}$ reaction, overshooting, thermal pulses, and mass loss, introduce uncertainties of the order of $1 \mathrm{~s}$ in the theoretical periods, so differences in $\phi \simeq 1 \mathrm{~s}$ represent good agreement (e.g., Bischoff-Kim et al. 2008). The model has not crystallized at this temperature. It starts to crystallize at $T_{\text {eff }} \simeq 10,500 \mathrm{~K}$ if we use the spindle diagram of Segretain \& Chabrier (1993) or at $T_{\text {eff }} \simeq 9600 \mathrm{~K}$ if we use the azeotropic phase diagram of Horowitz et al. (2010; see also Althaus et al. 2012).

We also used the simpler models described in Castanheira $\&$ Kepler (2008), which assume $\mathrm{C} / \mathrm{O}=50 \%$, but allow the helium and the hydrogen layer mass to vary, to do another seismological fit, with the results shown in Table 3. From the averages of the minima of various families of solutions, we obtained, $T_{\text {eff }}=11,900 \pm 200 \mathrm{~K}, M_{*}=0.88 \pm 0.08 M_{\odot}$, $M_{\mathrm{H}}=10^{-4.5 \pm 0.4} M_{*}$, and $M_{\mathrm{He}}=10^{-2.3 \pm 0.5} M_{*}$. The large difference in $M_{\mathrm{H}}$ of the two results may be due to the coreenvelope symmetry studied by Montgomery et al. (2003) and the simple C/O profile used in Castanheira \& Kepler (2008) models.

To assure that the prewhitening was not introducing uncertainties in the seismological solution, one of us independently measured the periodicities in the observed light curve by prewhitening; he obtained a period of $831 \mathrm{~s}$ for the smallest amplitude period measured, and measured another periodicity, at $525 \mathrm{~s}$, with an amplitude of $3.6 \pm 0.7 \mathrm{mma}$. The uncertainties quoted for the periods are the internal uncertainties of the fits; they do not take into account the possibility that the selected period is a sidelobe of the real period, caused by the presence of gaps in the data. For the low amplitude modes, selection of which sidelobe to prewhiten is sometimes ambiguous. We searched for independent seismological solutions but it resulted in the same model quoted above, with the smallest amplitude 

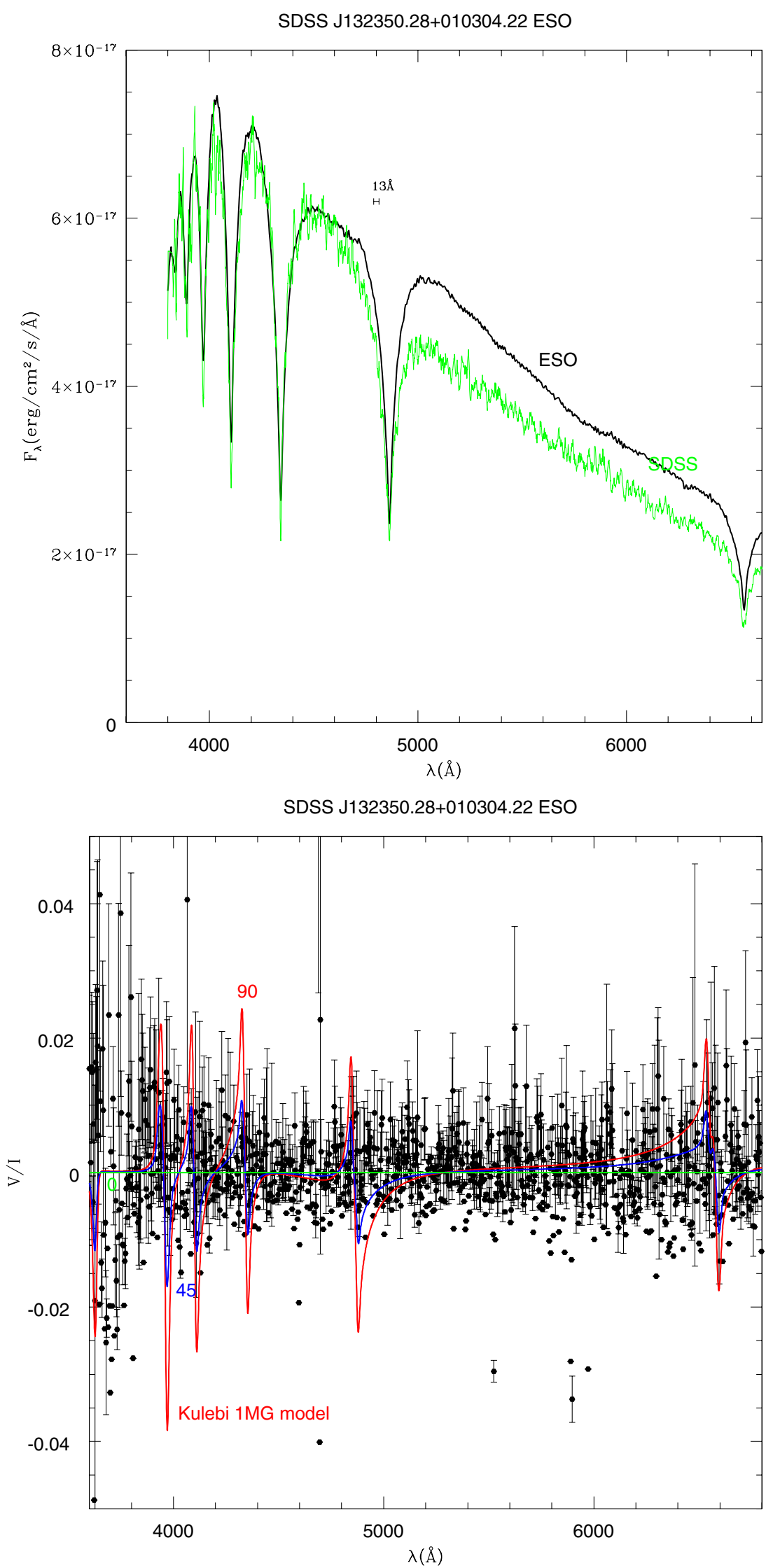

Figure 5. Spectropolarimetric measurements from ESO's VLT $8.2 \mathrm{~m}$ telescope. The upper plot shows the flux spectra, while the lower plot shows the observed $V / I$, compared to expected circular polarization for a $1 \mathrm{MG}$ field, at viewing angles of $0 \mathrm{deg}$ (pole on the line of sight), $45 \mathrm{deg}$, and $90 \mathrm{deg}$ (Külebi et al. 2009). (A color version of this figure is available in the online journal.) 


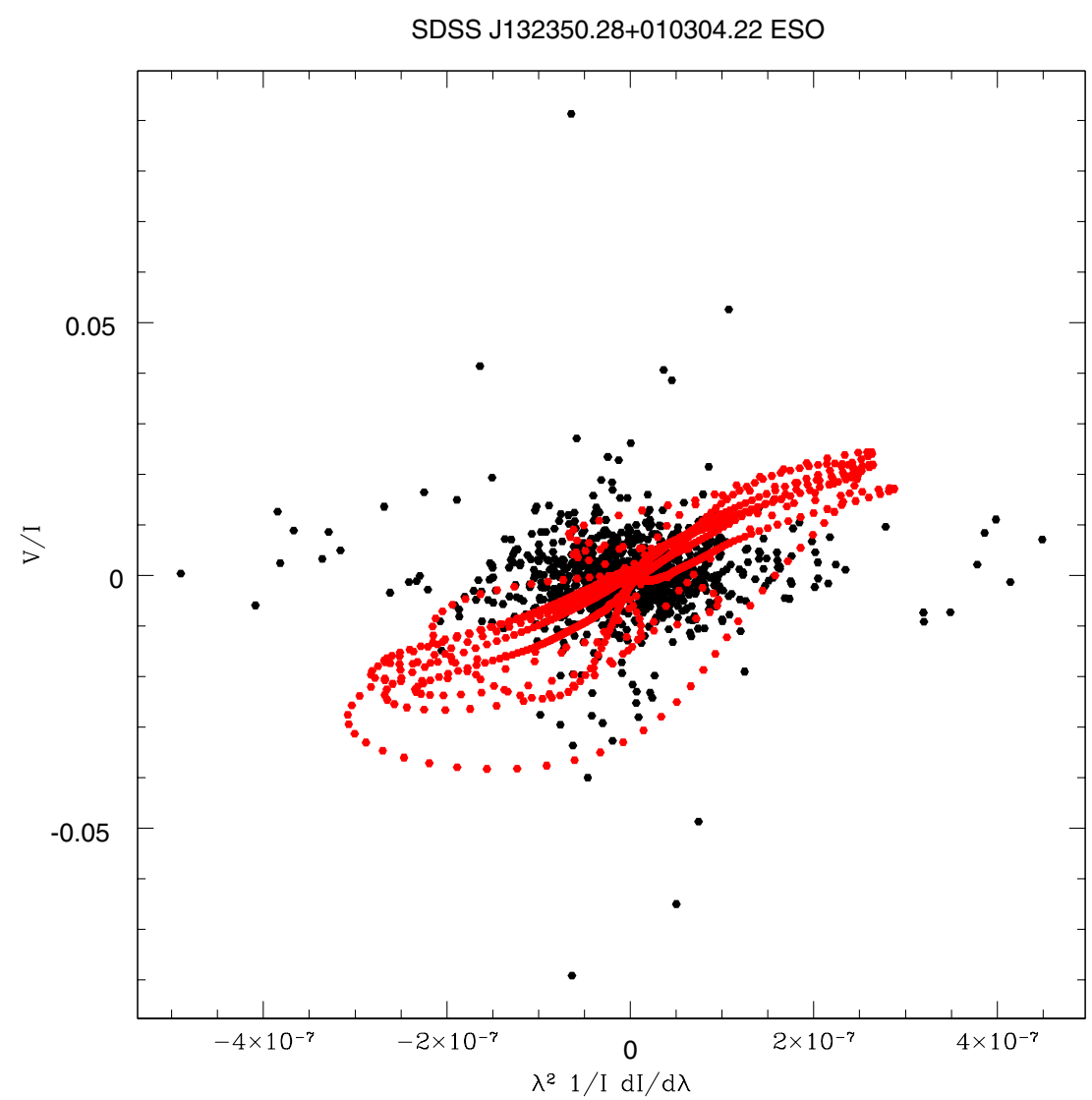

Figure 6. Circular polarization Stokes parameters $V / I$ plotted vs. $\lambda^{2}(1 / I)(d I / d \lambda)$, so the slope of the data points is directly the magnetic field (Bagnulo et al. 2002). For magnetic fields smaller than $B \simeq 300 \mathrm{kG}$, all the Zeeman components are linear in relation to this parameter. The red points show a 1 MG model. For such a field, the higher Balmer lines should show significant quadratic contributions, as $\Delta \lambda_{Q} \propto n^{4}$.

(A color version of this figure is available in the online journal.)

Table 3

Seismological Fit Following Castanheira \& Kepler (2008), C/O = 50\%

\begin{tabular}{|c|c|c|c|c|c|}
\hline$T_{\text {eff }}$ & $M$ & $-\log M_{\mathrm{H}}$ & $-\log M_{\mathrm{He}}$ & $\Phi(\mathrm{s})$ & Modes $(\ell, k)$ \\
\hline 11,800 & 0.84 & 4 & 2 & 2.7 & $\begin{array}{l}\text { 485.12(1,10), 550.03(1,12), 583.76(1,13), } \\
612.69(1,14), 642.75(1,15), 675.10(1,16), \\
705.57(1,17), 834.17(1,21), 897.42(1,23)\end{array}$ \\
\hline 11,700 & 0.88 & 4.5 & 2 & 2.9 & $\begin{array}{l}482.73(1,11), 550.25(1,13), 582.94(1,14), \\
612.21(1,15), 642.98(1,16), 673.45(1,17), \\
707.15(1,18), 833.59(1,22), 897.18(1,24)\end{array}$ \\
\hline 12,200 & 0.93 & 5 & 3 & 2.8 & $\begin{array}{l}489.20(1,11), 549.50(1,13), 585.51(1,14) \\
613.20(1,15), 643.45(1,16), 678.86(1,17), \\
710.05(1,18), 834.98(1,22), 897.13(1,24)\end{array}$ \\
\hline
\end{tabular}

modes fitting the model periodicities of $832.59 \mathrm{~s}$ and $534.47 \mathrm{~s}$. Therefore, we conclude that the detected periodicities and the seismological solution are robust.

\section{DISCUSSION}

Dolez \& Vauclair (1981) and Winget et al. (1982) demonstrated that the surface partial ionization drives the observed pulsations in ZZ Ceti stars, and Brickhill (1990) demonstrated that convective driving is important.

Schmidt \& Grauer (1997) and Valyavin et al. (2006) established only upper limits of a few $\mathrm{kG}$ for the handful of pulsating white dwarf stars they observed with spectropolarimetry. There has been no direct spectroscopic, spectropolarimetric, or polarimetric evidence for magnetic fields, of any strength, in the DBV or DAV stars. Fontaine et al. (1973) estimated that equipartition between magnetic energy and convection kinetic energy occurs for field strengths in the range 10-100 kG. Furthermore, applying the formalism of Gough \& Tayler (1966), we expect magnetic fields with vertical components in this range to effectively suppress convection.

SDSS J132350.28+010304.22 has a mass higher than the average mass of DAs (Kepler et al. 2007; Tremblay et al. 2010), has an effective temperature close the blue edge of the ZZ Ceti instability strip, even though the blue edge is mass dependent, and has long pulsation periods. Kepler et al. (1983), Handler et al. (2002), and Dolez et al. (2006), for example, used rotational splittings observed in some ZZ Ceti stars to estimate rotation periods of order of 1 day. We do not detect multiplets from rotational or magnetic splitting in this star, but such detections require longer and denser observations than reported here. 
The seismological mass agrees with the spectral determination, indicating a mass close to $0.88-0.9 M_{\odot}$. Comparing the SDSS colors to the theoretical photometry of Holberg \& Bergeron (2006) results in $T_{\text {eff }}=12,330 \pm 160 \mathrm{~K}$ and $\log g=8.13 \pm 0.22$. The relatively low $\log g$ from the colors is incompatible with the spectroscopic and seismological results, but its large uncertainty prevent its effective use.

The initial-to-final mass relation of Williams et al. (2009) indicates a mass of the progenitor of $(4.17 \pm 0.25) M_{\odot}$, consistent with the seismological progenitor mass.

The lack of reported magnetic fields among the 148 known ZZ Ceti stars suggests the possibility that there may be a difference in the ratio of magnetic to non-magnetic stars in and out of the instability strip. This makes it important to investigate if magnetic fields have been missed in the known DAVs or if there is an observational effect selecting against them.

This work is based on observations at the SOuthern Astrophysical Research telescope, a collaboration between CNPqBrazil, NOAO, UNC, and MSU, and on observations made with the ESO VLT telescopes at the Paranal Observatory under program ID 287.D-5037(A). SOAR proposals SO2011A002, SO2011A-007, and SO2011A-027. ESO proposal 287.D5037(A). S.O.K., J.E.S.C., I.P., and V.P. are supported by CNPq and FAPERGS/Pronex. D.E.W. and M.H.M. gratefully acknowledge the support of the US National Science Foundation under grant AST-0909107 and the Norman Hackerman Advanced Research Program under grant 003658-0252-2009. B.K. is supported by the MICINN grant AYA08-1839/ESP, by the ESF EUROCORES Program EuroGENESIS (MICINN grant EUI2009-04170), by the 2009SGR315 of the Generalitat de Catalunya and EU-FEDER funds. A.K. is supported by CNPq. A.H.C., A.D.R., and L.G.A. acknowledge AGENCIA through the Programa de Modernización Tecnológica BID 1728/OC-AR, and the PIP 112-200801-00940 grant from CONICET.

\section{REFERENCES}

Althaus, L. G., Córsico, A. H., Bischoff-Kim, A., et al. 2010a, ApJ, 717, 897 Althaus, L. G., Córsico, A. H., Isern, J., \& García-Berro, E. 2010b, A\&AR, 18, 471
Althaus, L. G., García-Berro, E., Isern, J., Córsico, A. H., \& Miller Bertolami, M. M. 2012, A\&A, 537, A33

Bagnulo, S., Landstreet, J. D., Fossati, L., \& Kochukhov, O. 2012, A\&A, 538, A129

Bagnulo, S., Szeifert, T., Wade, G. A., Landstreet, J. D., \& Mathys, G. 2002, A\&A, 389, 191

Bergeron, P., Wesemael, F., Dufour, P., et al. 2011, ApJ, 737, 28

Bergeron, P., Wesemael, F., Lamontagne, R., et al. 1995, ApJ, 449, 258

Bischoff-Kim, A., Montgomery, M. H., \& Winget, D. E. 2008, ApJ, 675, 1505

Brickhill, A. J. 1990, MNRAS, 246, 510

Castanheira, B. G., \& Kepler, S. O. 2008, MNRAS, 385, 430

Castanheira, B. G., Kepler, S. O., Kleinman, S. J., Nitta, A., \& Fraga, L. 2010, MNRAS, 405, 2561

Costa, J. E. S., Kepler, S. O., \& Winget, D. E. 1999, ApJ, 522, 973

Dolez, N., \& Vauclair, G. 1981, A\&A, 102, 375

Dolez, N., Vauclair, G., Kleinman, S. J., et al. 2006, A\&A, 446, 237

Fontaine, G., \& Brassard, P. 2008, PASP, 120, 1043

Fontaine, G., Thomas, J. H., \& van Horn, H. M. 1973, ApJ, 184, 911

Gough, D. O., \& Tayler, R. J. 1966, MNRAS, 133, 85

Handler, G., Romero-Colmenero, E., \& Montgomery, M. H. 2002, MNRAS, 335,399

Holberg, J. B., \& Bergeron, P. 2006, AJ, 132, 1221

Horowitz, C. J., Schneider, A. S., \& Berry, D. K. 2010, Phys. Rev. Lett., 104, 231101

Isern, J., García-Berro, E., Althaus, L. G., \& Córsico, A. H. 2010, A\&A, 512, A86

Kanaan, A., O’Donoghue, D., Kleinman, S. J., et al. 2000, Balt. Astron., 9, 387

Kepler, S. O., Costa, J. E. S., Castanheira, B. G., et al. 2005, ApJ, 634, 1311

Kepler, S. O., Kleinman, S. J., Nitta, A., et al. 2007, MNRAS, 375, 1315

Kepler, S. O., Robinson, E. L., \& Nather, R. E. 1983, ApJ, 271, 744

Koester, D. 2010, Mem. Soc. Astron. Ital., 81, 921

Koester, D., Kepler, S. O., Kleinman, S. J., \& Nitta, A. 2009, J. Phys.: Conf. Ser., 172, 012006

Külebi, B., Jordan, S., Euchner, F., Gänsicke, B. T., \& Hirsch, H. 2009, A\&A, 506,1341

Liebert, J., Bergeron, P., \& Holberg, J. B. 2003, AJ, 125, 348

Montgomery, M. H., Metcalfe, T. S., \& Winget, D. E. 2003, MNRAS, 344, 657 Podsiadlowski, P., Langer, N., Poelarends, A. J. T., et al. 2004, ApJ, 612, 1044

Romero, A. D., Córsico, A. H., Althaus, L. G., et al. 2012, MNRAS, 420, 1462

Schmidt, G. D., \& Grauer, A. D. 1997, ApJ, 488, 827

Schmidt, G. D., \& Smith, P. S. 1995, ApJ, 448, 305

Segretain, L., \& Chabrier, G. 1993, A\&A, 271, L13

Tremblay, P.-E., Bergeron, P., \& Gianninas, A. 2011, ApJ, 730, 128

Tremblay, P.-E., Bergeron, P., Kalirai, J. S., \& Gianninas, A. 2010, ApJ, 712, 1345

Valyavin, G., Bagnulo, S., Fabrika, S., et al. 2006, ApJ, 648, 559

Weidemann, V. 2000, A\&A, 363, 647

Wickramasinghe, D., \& Ferrario, L. 2005, MNRAS, 356, 1576

Williams, K. A., Bolte, M., \& Koester, D. 2009, ApJ, 693, 355

Winget, D. E., \& Kepler, S. O. 2008, ARA\&A, 46, 157

Winget, D. E., van Horn, H. M., Tassoul, M., et al. 1982, ApJ, 252, L65 